\title{
Caracterização do Perfil das Mulheres com Resultado Citológico ASCUS/AGC, LSIL e HSIL segundo Fatores Sociodemográficos, Epidemiológicos e Reprodutivos em Rio Branco - AC, Brasil
} Characterizing the Profile of Women with Cytological Results ASCUS/AGC, LSIL e HSIL, according to Socio-demographic, Epidemiological and Reproductive Factors in Rio Branco - AC, Brazil

Caracterización del Perfil de las Mujeres con Resultados Citológicos ASCUS/ AGC, LSIL Y HSIL según los Factores Sociodemográficos, Epidemiológicos y Reproductivos en Río Branco - AC, Brasil

Patrícia Rezende do Prado'; Rosalina Jorge Koifman²; Anna Luiza Moreira Santana ${ }^{3}$ ilce Ferreira da Silva ${ }^{4}$

\section{Resumo}

Introduçáo: $\mathrm{O}$ câncer do colo do útero é a segunda neoplasia mais incidente entre as mulheres. Na Regiáo Norte do Brasil, especialmente na cidade de Rio Branco/Acre, é o tipo de câncer com maior incidência e mortalidade. Objetivo: Caracterizar o perfil das mulheres rastreadas com resultados colpocitológicos de ASCUS/AGC, LSIL e HSIL, entre 2007 a 2008, no setor público da na cidade de Rio Branco, AC. Método: Estudo transversal, com mulheres diagnosticadas com resultados colpocitológicos de ASCUS/AGUS, LSIL e HSIL, identificadas através do exame Papanicolaou e registradas no Programa de Controle de Câncer do Colo do Útero nos anos de 2007 e 2008 no Centro de Controle em Oncologia do Acre (CECON/AC). Resultados: 22,6\% das mulheres tinham menos que 25 anos de idade, 57,3\% tinham entre 25 a 45 anos, 89,1\% eram pardas, 59,8\% tinham o nível fundamental de escolaridade, 30,5\% tiveram a sexarca antes dos 14 anos, $64,9 \%$ tinham acima de 3 filhos, 70,0\% tiveram mais que 2 parceiros ao longo da vida, $54,0 \%$ não havia recebido tratamento após o diagnóstico citológico alterado e 45,7\% dos diagnósticos citológicos de atipias de significado indeterminado eram lesôes de alto grau ou câncer no histopatológico. Conclusáo: Sugere-se que sejam implementadas melhorias na qualidade do programa de prevençấo do câncer do colo do útero do Estado do Acre, envolvendo a avaliação, controle do diagnóstico e tratamento, especialmente das mulheres diagnosticadas como ASCUS/AGC, para que se possa esperar alguma redução nas taxas de incidência e mortalidade por câncer do colo do útero nessa Região.

Palavras-chave: Neoplasias do Colo do Útero/prevenção \& controle; Neoplasias do Colo do Útero/terapia; Programas de Rastreamento; Estudos Transversais

\footnotetext{
Apoio: Pesquisa financiada parcialmente pela Fundaçáo de Tecnologia do Estado do Acre (FUNTAC). ${ }^{1}$ Bolsista de produtividade durante o mestrado pelo CNPq.

${ }^{1}$ Enfermeira. Mestranda do Programa de Pós-Graduaçâo em Saúde Coletiva pela Universidade Federal do Acre. Rio Branco (AC), Brasil.

${ }^{2}$ Médica. Doutora do Departamento de Pós-Graduação em Saúde Pública e Meio Ambiente da Escola Nacional de Saúde Pública Sergio Arouca. Fundação Oswaldo Cruz. Rio de Janeiro (RJ), Brasil.

${ }^{3}$ Acadêmica de Enfermagem da Universidade Federal do Acre.

${ }^{4}$ Enfermeira. Doutora do Departamento de Epidemiologia da Universidade Federal Fluminense (UFF). Rio de Janeiro (RJ), Brasil.

Endereço para correspondência: Patricia Rezende do Prado. Alameda Atenas, 67, apto 93, Condomínio Florença, Ed. San Marino. Jardim Europa II. Rio Branco (AC), Brasil. CEP: 69911-140.E-mail: patyrezende@terra.com.br.
} 


\section{INTRODUÇÃO}

O câncer do colo do útero é a segunda neoplasia mais incidente entre as mulheres, responsável por $15 \%$ dos cânceres femininos, com aproximadamente 500 mil casos novos e 230 mil mortes por ano no mundo. $\mathrm{Na}$ América Latina e Sudeste Asiático, as taxas de incidência são geralmente elevadas, enquanto na América do Norte, Austrália, Norte e Oeste Europeu, são consideradas baixas $^{1-2}$.

No Brasil, o câncer do colo do útero apresenta diferentes distribuiçóes entre as regióes brasileiras, sendo a primeira causa de mortalidade na Região Norte com 24/100.000 mulheres, enquanto a Região Sudeste ocupa a quinta posição com 15/100.000. Em 2006, a magnitude das taxas de mortalidade da Regiáo Norte foi o dobro das taxas da Região Sul em todas as faixas etárias, no mesmo período ${ }^{3}$.

Em um estudo sobre a "Tendência da mortalidade por neoplasias malignas em Rio Branco, Acre”, foi relatado que o câncer do colo do útero representou a neoplasia com maiores taxas de incidência $(36,7 / 100.000)$ e mortalidade $(10,7 / 100.000)$ em $2009^{4}$.

A infecção por subtipos oncogênicos do Papilomavírus humano (HPV) é considerada o fator causal primário para o desenvolvimento da neoplasia invasora do colo do útero e esse vírus é adquirido através de transmissão sexual ${ }^{5-6}$. Outros fatores como a idade, cor, herança genética, idade da sexarca, número de parceiros sexuais, número de gestações, hábitos alimentares, tabagismo, outros agentes infecciosos e situação socioeconômica são fatores considerados de risco associados ao desenvolvimento do câncer do colo do útero. Essa exposição é cumulativa no tempo e, portanto, o risco de desenvolver câncer aumenta com a idade, porém, essa neoplasia pode ser prevenida por meio do exame preventivo de Papanicolaou 5 .

O exame citológico do Papanicolaou detecta as alterações do colo do útero sugerindo alterações intraepiteliais das células escamosas, recebendo a classificação de neoplasia intraepitelial grau I (NIC I), quando atinge a camada basal do epitélio, neoplasia intraepitelial grau II (NIC II) quando avança até três quartos da espessura do epitélio, neoplasia intraepitelial cervical grau III (NIC III) quando atinge todas as camadas e carcinoma invasor quando as alteraçôes são mais intensas e invadem o tecido conjuntivo abaixo do epitélio ${ }^{1,5}$. Em 1988, o National Cancer Institute nos EUA propôs um novo esquema de registro dos esultados da citologia cervical, que mais tarde se tornou as lesôes de baixo grau (LSIL), compreendendo as alteraçōes por HPV e NIC-I, e as lesôes de alto grau (HSIL) que compreendem as NICII e III. As alteraçôes que não podiam ser classificadas em lesôes de baixo ou alto grau foram classificadas em atipias de significado indeterminado de células escamosas (ASCUS) ou de células glandulares (AGC) ${ }^{6-7}$.

Em Rio Branco, um inquérito de base populacional realizado em 2007-2008 revelou uma expressiva cobertura do exame preventivo chegando a $85,3 \%$ na população-alvo ${ }^{8}$.

Com a finalidade de subsidiar a compreensão da situação do câncer do colo do útero em Rio Branco, Acre, possibilitando o levantamento de novas hipóteses, foi desenvolvido este estudo seccional com o objetivo de caracterizar o perfil das mulheres rastreadas com resultados colpocitológicos de ASCUS/AGC, LSIL e HSIL, entre 2007 a 2008, no setor público da na cidade de Rio Branco (AC), Brasil.

\section{MÉTODO}

Estudo observacional do tipo transversal realizado com mulheres diagnosticadas com resultados colpocitológicos de ASCUS/AGC, LSIL e HSIL, identificadas por meio do teste de Papanicolaou e registradas no Programa de Controle de Câncer do Colo do Útero (PCCCU/ SISCOLO) nos anos de 2007 e 2008 na cidade de Rio Branco (AC), Brasil.

Após a seleção das mulheres com resultados colpocitológicos de ASCUS/AGC, LSIL e HSIL atendidas no período de 2007 e 2008 , foram selecionados os prontuários das pacientes que foram atendidas no Centro de Controle Oncológico do Acre (Cecon) e nas unidades básicas de saúde. A identificação do prontuário foi realizada pelo nome, data de nascimento, nome da máe da paciente e número do exame alterado. A extração dos dados foi realizada em formulário padronizado, elaborado para esse fim.

Foram coletadas variáveis sociodemográficas (idade, naturalidade, estado civil, cor, escolaridade), epidemiológicas (tabagismo, menarca, sexarca, gestação, número de parceiros, uso de contraceptivo oral) e clínicas (citologia de entrada, resultado da primeira histologia).

A variável escolaridade foi classificada em analfabeta/ anos de estudo; o tabagismo foi classificado como nunca fumou/ex-tabagista/tabagismo atual, e o resultado citopatológico e histológico seguiram a classificação do Sistema Bethesda, 2001.

$\mathrm{Na}$ análise estatística, as diferenças entre as variáveis contínuas foram analisadas por medidas de tendência central e dispersão. As diferenças entre as variáveis categóricas foram avaliadas por estimativas das proporçóes de cada variável, segundo o grau de lesão, usando o teste qui-quadrado. Para todas as análises, foi considerado um grau de significância de 5\%. Os dados foram digitados no Excel 2010 e analisado no SPSS 13.0. 
Este projeto foi aprovado pelo Comitê de Ética em Pesquisa da Universidade Federal do Acre (UFAC) sob o $\mathrm{n}^{\mathrm{o}}$ de Protocolo 23107. 016700/2010-11.

\section{RESULTADOS}

Entre os 48.729 exames colpocitológicos realizados pelo programa de rastreamento do câncer do colo do útero do município de Rio Branco (AC), Brasil, nos anos de 2007 e 2008, 846 (1,74\%) mulheres do setor público de saúde apresentaram resultados colpocitológicos de ASCUS/AGC, LSIL e HSIL no exame de Papanicolaou (Tabela 1).

Quando comparados os anos de 2007 e 2008 (Tabela 1), foi observado que a proporção de exames alterados é semelhante nos dois anos estudados (2,1\% em 2007 e 1,5\% em 2008). No entanto, lesão intraepitelial de alto grau (HSIL) e câncer ocorreram com uma maior frequência em 2007 (0,30\% e 0,13\%, respectivamente) quando comparadas ao ano de $2008(0,18 \%$ e $0,02 \%$, respectivamente).

Tabela 1. Distribuição dos resultados citológicos de 2007 e 2008 nas mulheres de Rio Branco (AC), Brasil

\begin{tabular}{l|c|c|c|c}
\hline \multirow{2}{*}{ CITOLOGIA } & \multicolumn{2}{|c|}{2007} & \multicolumn{2}{c}{2008} \\
\cline { 2 - 5 } & $\mathbf{N}$ & $\mathbf{( \% )}$ & $\mathbf{N}$ & $\mathbf{( \% )}$ \\
\hline Negativa & 20.571 & 97,9 & 27.312 & 98,48 \\
\hline LSIL & 74 & 0,35 & 80 & 0,29 \\
\hline HSIL & 63 & 0,30 & 49 & 0,18 \\
\hline ASCUS/AGC & 276 & 1,32 & 287 & 1,03 \\
\hline Câncer & 10 & 0,13 & 7 & 0,02 \\
\hline TOTAL & 20.994 & 100 & 27.735 & 100 \\
\hline
\end{tabular}

LSIL: Lesão intraepitelial de baixo grau. HSIL: Lesão intraepitelial de alto grau

Entre as mulheres com resultados colpocitológicos de ASCUS/AGC, LSIL e HSIL, 191 (22,6\%) tinham menos de 25 anos e 485 (57,3\%) tinham entre 25 e 45 anos. A maioria $(96,8 \%)$ dessas mulheres era natural do Estado do Acre, $89,1 \%$ eram pardas e $59,8 \%$ haviam estudado até o ensino fundamental (Tabela 2).

Cerca de metade dessas mulheres náo tinham companheiro $(50,8 \%)$ e $70 \%$ tiveram mais de dois parceiros sexuais ao longo da vida. A menarca ocorreu até os 12 anos de idade em 13,2\% das mulheres, $47,6 \%$ entre 12 e 13 anos. A sexarca ocorreu antes dos 14 anos de idade em $30,5 \%$, enquanto em $50,8 \%$ ocorreu entre 15 a 17 anos. A maioria das mulheres (64,9\%) tinha acima de três filhos na data do rastreamento. Em relaçáo ao hábito de fumar, $36 \%$ eram fumantes e $8,3 \%$ eram ex-fumantes (Tabela 2).
Tabela 2. Caracterização das mulheres com alterações citológicas, Rio Branco, 2007 e 2008

\begin{tabular}{|c|c|c|c|}
\hline VARIÁVEL & $\mathbf{N}^{*}$ & $\%$ & IC $95 \%$ \\
\hline \multicolumn{4}{|l|}{ IDADE } \\
\hline$<25$ anos & 191 & 22,6 & $19,8-25,4$ \\
\hline $25-45$ anos & 485 & 57,3 & $54,0-60,0$ \\
\hline$>45$ anos & 170 & 20,1 & $17,4-22,8$ \\
\hline \multicolumn{4}{|l|}{ NATURALIDADE } \\
\hline Acreana & 819 & 96,8 & $95,6-98,0$ \\
\hline Não acreana & 27 & 3,2 & $2,0-4,0$ \\
\hline \multicolumn{4}{|l|}{ ESTADO CIVIL } \\
\hline Com companheiro & 214 & 49,2 & $44,5-53,9$ \\
\hline Sem companheiro & 221 & 50,8 & $46,1-55,5$ \\
\hline \multicolumn{4}{|l|}{ COR } \\
\hline Branca & 24 & 6,5 & $4,0-9,1$ \\
\hline Negra & 14 & 3,8 & $1,9-5,8$ \\
\hline Amarela & 2 & 0,5 & - \\
\hline Parda & 327 & 89,1 & $85,9-92,3$ \\
\hline \multicolumn{4}{|l|}{ ESCOLARIDADE } \\
\hline Não estudou & 34 & 12,3 & $8,4-16,1$ \\
\hline Até $1^{\circ}$ grau & 166 & 59,8 & $54,2-65,7$ \\
\hline$>1^{\circ}$ grau & 77 & 27,8 & $22,5-33,1$ \\
\hline \multicolumn{4}{|l|}{ FUMO } \\
\hline Nunca fumou & 155 & 55,8 & $49,9-61,6$ \\
\hline Ex-fumante & 23 & 8,3 & $5,0-11,5$ \\
\hline Fumante atual & 100 & 36,0 & $30,3-41,6$ \\
\hline \multicolumn{4}{|l|}{ MENARCA } \\
\hline$<12$ anos & 55 & 13,2 & $10,0-16,5$ \\
\hline $12-13$ anos & 198 & 47,6 & $42,8-52,4$ \\
\hline$>14$ anos & 163 & 39,2 & $34,5-43,9$ \\
\hline \multicolumn{4}{|l|}{ SEXARCA } \\
\hline$<14$ anos & 119 & 30,5 & $25,9-35,1$ \\
\hline $15-17$ anos & 198 & 50,8 & $49,8-55,7$ \\
\hline$>18$ anos & 73 & 18,7 & $14,8-22,6$ \\
\hline \multicolumn{4}{|l|}{ GESTAÇÃO } \\
\hline Nunca & 7 & 1,6 & $0,4-2,8$ \\
\hline 1 - 2 gestações & 144 & 33,5 & $29,0-37,9$ \\
\hline 3 - 4 gestações & 130 & 30,2 & $25,9-34,6$ \\
\hline$>5$ gestações & 149 & 34,7 & $30,2-39,1$ \\
\hline \multicolumn{4}{|l|}{ PARCEIROS } \\
\hline 1 parceiro & 108 & 30,0 & $25,0-34,0$ \\
\hline 2 - 3 parceiros & 165 & 45,8 & $40,0-51,0$ \\
\hline$>4$ parceiros & 87 & 24,2 & $20,0-29,0$ \\
\hline \multicolumn{4}{|l|}{ Resultado citologia } \\
\hline LSIL & 154 & 18,2 & $15,6-20,8$ \\
\hline HSIL & 112 & 13,2 & $11,0-15,0$ \\
\hline ASCUS/AGC & 563 & 66,5 & $63,4-70,0$ \\
\hline Câncer & 17 & 2,0 & $11,0-30,0$ \\
\hline \multicolumn{4}{|l|}{$\begin{array}{l}\text { Qualidade da } \\
\text { amostra }\end{array}$} \\
\hline Satisfatória & 846 & 100,0 & - \\
\hline
\end{tabular}

${ }^{*}$ Os valores podem alterar devido aos missings

LSIL: Lesão intraepitelial de baixo grau. HSIL: Lesão intraepitelial de alto grau AGC: célula glandular atípica de significado indeterminado 
Em relação aos resultados colpocitológicos de ASCUS/ AGC, LSIL e HSIL, 18,2\% ( $\mathrm{n}=154)$ apresentaram lesão intraepitelial de baixo grau (LSIL), 13,2\% ( $\mathrm{n}=112)$ lesão intraepitelial de alto grau (HSIL), 66,5\% ( $\mathrm{n}=563)$ atipias de significado indeterminado (ASCUS/AGC e 2,0\% ( $\mathrm{n}=17)$ tinham câncer (Tabela 2).

Quanto à conduta implementada, 54,0\% das mulheres com resultados colpocitológicos de ASCUS/AGC, LSIL e HSIL não receberam tratamento. Dentre essas, 20,4\% apresentavam resultado citopatológico de LSIL, 7,0\% apresentavam HSIL, 72,0\% eram ASCUS/AGC e 0,6\% tinham citopatologia compatível com câncer (Tabela 3).

Em relação à conduta terapêutica, das 274 mulheres tratadas, cinco não realizaram biópsia e, portanto, não têm resultado histopatológico, o que explica o total de 269 e não 274 mulheres.

A cirurgia de alta frequência (CAF) / conização foi o tratamento implementado em $100 \%$ das mulheres com LSIL (NIC I, HPV) e HSIL (NIC II) que foram tratadas, além de $93,8 \%$ das NIC-III e $42,1 \%$ dos casos de câncer. A histerectomia foi implementada em 6,2\% das mulheres com NIC-III e $10,5 \%$ das que tiveram câncer. $\mathrm{O}$ tratamento clínico (radio e quimioterapia) foi a opção de tratamento para $47,4 \%$ das mulheres com câncer (Tabela 4).
Na Tabela 5, verifica-se que 33,3\% das mulheres com citopatologia de LSIL apresentaram resultado histológico negativo, enquanto $50,0 \%$ realmente eram NIC I, e $16,7 \%$ eram NIC II/III. Entre as mulheres com citologia de HSIL, 16,3\% eram negativas e 20,0\% apresentavam câncer no laudo histopatológico. Dentre as Atipias, 33,6\% eram negativas, $35,7 \%$ NIC II/III e 10,0\% câncer. Já as mulheres com resultado citopatológico de câncer, $15,4 \%$ eram negativas no histopatológico, 30,8\% NIC II/III e 53,8\% apresentavam câncer.

\section{DISCUSSÃO}

O presente estudo mostra que os resultados colpocitológicos de ASCUS/AGC, LSIL e HSIL foram mais frequentes em mulheres entre 25 e 45 anos, pardas, com baixo grau de escolaridade, maior número de parceiros sexuais, além da sexarca precoce, multíparas e tabagistas, corroborando com os achados dos estudos desenvolvidos no Brasil e em países em desenvolvimento ${ }^{9-11}$.

No entanto, mesmo os estudos conduzidos em países desenvolvidos têm mostrado que os resultados colpocitológicos de ASCUS/AGC, LSIL e HSIL são mais frequentes em mulheres em situaçôes de vulnerabilidade social (baixa escolaridade, baixo nível socioeconômico,

Tabela 3. Tratamentos implementados nas mulheres de Rio Branco/AC, segundo resultado histológico no rastreamento, 2007-2008

\begin{tabular}{|c|c|c|c|c|c|c|c|c|}
\hline \multicolumn{9}{|c|}{ Tratamento implementado } \\
\hline \multirow{2}{*}{ Histologia de entrada } & \multicolumn{2}{|c|}{$\begin{array}{l}\text { Exérese } \\
\text { da lesão }\end{array}$} & \multicolumn{2}{|c|}{ Histerectomia } & \multicolumn{2}{|c|}{ RT/QT } & \multicolumn{2}{|c|}{ Total } \\
\hline & $\mathbf{N}$ & $\%$ & $\mathbf{N}$ & $\%$ & $\mathbf{N}$ & $\%$ & $\mathbf{N}$ & $\%$ \\
\hline HPV, Metaplasia & 74 & 100,0 & - & - & - & - & 74 & 100 \\
\hline $\mathrm{NICl}$ & 53 & 100,0 & - & - & - & - & 53 & 100 \\
\hline NIC II & 40 & 100,0 & - & - & - & - & 40 & 100 \\
\hline NIC III & 60 & 93,8 & 4 & 6,2 & - & - & 64 & 100 \\
\hline Câncer & $16^{*}$ & 42,1 & 4 & 10,5 & 18 & 47,4 & 38 & 100 \\
\hline Total & 243 & 90,3 & 8 & 3,0 & 18 & 6,7 & 269 & 100 \\
\hline
\end{tabular}

Tabela 4. Conduta implementada nas mulheres com citologias alteradas em Rio Branco/AC, 2007-2008

\begin{tabular}{|c|c|c|c|c|c|c|c|c|}
\hline \multirow[t]{2}{*}{ Citologia entrada } & \multicolumn{2}{|c|}{ Não tratadas } & \multicolumn{2}{|c|}{ Tratadas } & \multicolumn{2}{|c|}{$\begin{array}{l}\text { Tratamento } \\
\text { expectante }\end{array}$} & \multicolumn{2}{|c|}{ Total } \\
\hline & $\mathbf{N}$ & $\%$ & $\mathbf{N}$ & $\%$ & $\mathbf{N}$ & $\%$ & $\mathbf{N}$ & $\%$ \\
\hline LSIL & 93 & 60,4 & 36 & 23,4 & 25 & 16,2 & 154 & 100 \\
\hline HSIL & 32 & 28,6 & 80 & 71,4 & 0 & - & 112 & 100 \\
\hline ASCUS/AGC & 329 & 58,4 & 144 & 25,6 & 90 & 16,0 & 563 & 100 \\
\hline Câncer & 3 & 17,6 & 14 & 82,4 & 0 & - & 17 & 100 \\
\hline Total & 457 & 54,0 & 274 & 32,4 & 115 & 13,6 & 846 & 100 \\
\hline
\end{tabular}

* Citologia : 7 Atipias, 8 HSIL e 1 Câncer

LSIL: Lesão intraepitelial de baixo grau. HSIL: Lesão intraepitelial de alto grau

AGC: célula glandular atípica de significado indeterminado 
Tabela 5.Distribuição dos resultados histopatológicos pós-exérese da lesão, segundo o resultado colpocitológico nas mulheres de Rio Branco/ AC, 2007-2008

\begin{tabular}{|c|c|c|c|c|c|c|c|c|c|c|c|c|}
\hline \multirow{2}{*}{$\begin{array}{l}\text { Citologia } \\
\text { de entrada }\end{array}$} & \multicolumn{2}{|c|}{$\begin{array}{c}\text { HPV/ } \\
\text { Metaplasia }\end{array}$} & \multicolumn{2}{|c|}{ NIC I } & \multicolumn{2}{|c|}{ NIC II } & \multicolumn{2}{|c|}{ NIC III } & \multicolumn{2}{|c|}{ Câncer } & \multicolumn{2}{|c|}{ Total } \\
\hline & $\mathbf{N}$ & $\%$ & $\mathbf{N}$ & $\%$ & $\mathbf{N}$ & $\%$ & $\mathbf{N}$ & $\%$ & $\mathbf{N}$ & $\%$ & $\mathbf{N}$ & $\%$ \\
\hline LSIL & 12 & 33,3 & 18 & 50,0 & 4 & 11,1 & 2 & 5,6 & 0 & 0 & 36 & 100 \\
\hline HSIL & 13 & 16,3 & 5 & 6,3 & 17 & 21,3 & 29 & 36,3 & 16 & 20,0 & 80 & 100 \\
\hline ASCUS/AGC & 47 & 33,6 & 29 & 20,7 & 19 & 13,6 & 31 & 22,1 & 14 & 10,0 & 140 & 100 \\
\hline Câncer & 2 & 15,4 & 0 & 0,0 & 1 & 7,7 & 3 & 23,1 & 7 & 53,8 & 13 & 100 \\
\hline Total & 74 & 27,5 & 52 & 19,3 & 41 & 15,2 & 65 & 24,2 & 37 & 13,8 & 269 & 100 \\
\hline
\end{tabular}

LSIL: Lesão intraepitelial de baixo grau. HSIL: Lesão intraepitelial de alto grau AGC: célula glandular atípica de significado indeterminado

elevado número de filhos, tabagista, e geralmente imigrantes) $)^{11-12}$.

Em Rio Branco, no ano de 2001, foi realizado um estudo transversal que identificou que $6,9 \%$ das alteraçóes cervicais ocorreram em adolescentes de 15 a 19 anos sexualmente ativas ${ }^{10}$. Isto sugere que a exposição à infecção pelo HPV entre as mulheres de Rio Branco tem ocorrido muito precocemente.

Os achados do presente estudo corroboram essa hipótese, ao revelar que $93,0 \%$ das mulheres estudadas tiveram o primeiro intercurso sexual antes dos 17 anos de idade, enquanto $30,5 \%$ delas tiveram o primeiro intercurso sexual antes dos 14 anos. A sexarca precoce vem sendo associada ao risco de câncer do colo do útero, porque no período da puberdade e adolescência a zona de transformação cervical nessas mulheres jovens está localizada na ectocérvice, ficando mais exposta e vulnerável à infecção pelo HPV durante o intercurso sexual. Além disso, quanto mais precoce o início da atividade sexual, maior a oportunidade de múltiplos parceiros ao longo da vida e, consequentemente, maior a probabilidade de múltiplas infecçôes por diferentes tipos de $\mathrm{HPV}^{10}$.

No estudo multicêntrico denominado Latin America Screening (LAMS) ${ }^{11}$, envolvendo mulheres submetidas ao rastreamento para o câncer do colo do útero no Sistema Único de Saúde (SUS) das cidades de São Paulo, Campinas e Porto Alegre, foi observado que $20 \%$ da populaçáa estudada teve o primeiro intercurso sexual antes dos 15 anos de idade. Além disso, foi encontrado que quanto mais jovem a mulher, mais cedo a sexarca havia ocorrido. Neste mesmo estudo, foi observado que mulheres com idade da sexarca abaixo da média da amostra apresentaram maior positividade para HPV e maior número de alteraçóes citológicas, se comparadas às mulheres cuja sexarca tinha ocorrido em idade acima da média amostral.

Os achados de um estudo realizado em Montreal, no Canadá, dão suporte a essa hipótese, mostrando que $64 \%$ dos casais jovens com vida sexual recente (menor que 3,9 meses) tinham pelo menos um tipo de HPV, sendo que $41 \%$ tinham os mesmos tipos de HPV sugerindo alta transmissão. Entre os indivíduos positivos para HPV, $22 \%$ apresentavam $\mathrm{HPV}^{16}$, sendo este de alto risco para o câncer do colo do útero ${ }^{12}$.

Entre as mulheres com resultados colpocitológicos de ASCUS/AGC, LSIL e HSIL apresentadas neste estudo, $92,9 \%$ eram de cor da pele não branca. Em estudos nacionais e internacionais, o risco de desenvolvimento de lesóes do tipo ASCUS/AGC, LSIL e HSIL tem sido frequentemente associado à cor da pele parda e negra. No entanto, sabe-se que a cor está intrinsicamente relacionada ao baixo nível socioeconômico e ao acesso aos serviços de saúde?.

O baixo nível socioeconômico vem sendo associado a essa neoplasia, no entanto, isso pode indicar uma baixa qualidade das políticas públicas no controle do câncer na populaçáo de baixo nível socioeconômico ${ }^{13}$. No inquérito de base populacional realizado por Borges et al. ${ }^{8}$, foi observado que a maioria das mulheres (75\%) é atendida pelo Sistema Único de Saúde (SUS), sugerindo que esse serviço é o maior responsável pelo rastreamento do câncer do colo do útero na cidade de Rio Branco. Assim, torna-se necessário a melhor estruturação do programa de rastreamento do câncer do colo do útero para que haja convocaçôes dessas que dependem do sistema público de saúde para realizarem o diagnóstico precoce e um tratamento efetivo ${ }^{8}$.

O baixo nível de escolaridade, que geralmente é utilizado como um substituto do nível socioeconômico tem sido relatado como um fator de risco para o câncer do colo do útero, sugerindo que essas mulheres podem não reconhecer a importância do exame, ou não ter o conhecimento necessário para buscar rastreamento e tratamento, ou acesso ao serviço de saúde ${ }^{10}$. Em dois estudos transversais desenvolvidos em Santiago, no Chile, e na cidade do México, com o objetivo de investigar o conhecimento sobre o teste preventivo de Papanicolaou, 
foi observada que a deficiência de conhecimento sobre o exame preventivo é frequente em mulheres de baixa escolaridade e nível socioeconômico ${ }^{10}$.

O tabagismo tem sido relatado como um fator de risco independente para do câncer do colo do útero, uma vez que os carcinógenos presentes no cigarro atuam induzindo a formação de aductos, mutaçóes e promovendo a multiplicação desordenada das células infectadas pelo HPV. Existem estudos que indicam que os carcinógenos também atuariam promovendo a imunossupressã̃o ${ }^{14-17}$. Alteraçôes verificadas no sistema imune periférico de pacientes fumantes incluem a elevação do número de células sanguíneas, o aumento do número de linfócitos Tcitotóxicos/supressores, a diminuição do número de linfócitos $\mathrm{T}$ indutores/auxiliares, discreta supressão da atividade de linfócitos $\mathrm{T}$, significativo decréscimo da atividade de linfócitos natural killer, e baixos níveis sanguíneos de imunoglobulinas, exceto pela $\mathrm{IgE}$, que é elevada ${ }^{17}$. No presente estudo, a prevalência de tabagismo foi de $36 \%$, enquanto na populaçáo geral de Rio Branco essa prevalência é de $19 \%{ }^{16}$. Os dois mecanismos principais pelos quais o hábito de fumar contribui para a oncogênese cervical incluem a exposição direta do DNA de células epiteliais cervicais à nicotina, à cotidina e à imunossupressão ${ }^{17}$. Esse achado sugere que o tabagismo pode ser um cofator ambiental importante na carcinogênese cervical.

Um inquérito populacional realizado na cidade de Campinas/SP nos anos de 2001 e 2002 identificou que idade acima de 40 anos, mulheres negras ou mestiças, menos de quatro anos de escolaridade eram preditores de não realização do exame preventivo do câncer do colo do útero e os motivos eram achar desnecessário, constrangimento e dificuldades relacionadas ao acesso ao serviço de saúde ${ }^{18}$. Isto evidencia que os serviços devem promover acesso mais equitativo ao sistema de saúde, tomando as questóes ligadas à escolaridade e cor da pele como referência de acesso e nível socioeconômico ${ }^{19}$.

Quanto aos resultados das citologias, foi observado que, apesar da frequência de ASCUS/AGC estar dentro da recomendação do Sistema Bethesda, da Sociedade Americana de Colposcopia e do Ministério de Saúde Brasileiro, que orienta que menos de $5 \%$ das alteraçóes em um laboratório sejam de atipias, a frequência dessas atipias está acima do triplo da frequência de LSIL, o que não está de acordo com as recomendaçôes do sistema Bethesda, que preconiza que os resultados de ASCUS/AGC não devam ultrapassar o dobro das lesôes de baixo grau ${ }^{20}$.

A grande preocupação das categorias indeterminadas é ser usada como via de saída para limitaçôes pessoais para diagnosticar corretamente a alteraçáo citológica, erro frequente encontrado pela subjetividade do observador. Para evitar esse problema, os laboratórios devem ter mecanismos de controle de qualidade laboratorial internos checando aleatoriamente uma proporção de lâminas além de controlar o número de atipias e realizar capacitação e reciclagem de seus servidores ${ }^{21}$.

As atipias escamosas são dez vezes mais frequentes que as glandulares, porém, as glandulares oferecem maior risco para lesóes de alto grau e câncer. Aproximadamente 9 a $38 \%$ das mulheres com AGC apresentam NIC II e NIC III e adenocarcinoma in situ e 3 a $17 \%$ apresentam câncer ${ }^{22}$. Em nossos resultados, as atipias foram os resultados citológicos mais preocupantes, visto que $37,5 \%$ eram lesôes de alto grau e 10\% eram câncer na histologia, o que demonstra que os resultados citológicos não foram diagnosticados corretamente, postergando o tempo de tratamento das mulheres acreanas com lesóes cervicais. Esse resultado se torna mais preocupante ainda quando se verifica que apenas $41,6 \%$ das atipias foram tratadas e, portanto, $58,4 \%$ continuaram sem tratamento o que pode inferir que muitas acreanas com lesóes de alto grau e câncer estão sem tratamento.

Fatores como formação profissional, experiência, sobrecarga de trabalho, dificuldade diagnóstica entre outros, interferem na avaliaçáo dos casos de atipias de significado indeterminado deturpando a real importância e a função dessa classe. As taxas de atipias indeterminadas podem ser reduzidas se os profissionais que coletam as amostras realizarem com qualidade e correta fixação das lâminas. A representatividade das células da junção escamocolunar (JEC), a realização da coloração adequada pelo patologista, o diagnóstico preciso, além do controle interno de qualidade laboratorial ${ }^{23}$, são essenciais para o rastreamento do câncer do colo do útero seguro e eficaz.

Em estudo dos diagnósticos cito-histopatológicos das atipias de significado indeterminado do Instituto Adolfo Lutz, dos 60 casos de ASCUS com laudo histológico correspondente, $52,6 \%$ eram negativos, $28,1 \%$ eram NIC I, 10,53\% NIC II, 1,8\% NIC III e 7,0\% câncer ${ }^{24}$. A correlação cito-histopatológica tem sido apontada como um dos indicadores para medir a qualidade das citologias dos laboratórios de citologia do programa de prevenção do câncer do colo do útero.

A conduta orientada pela Sociedade Americana de Colposcopia é repetir a citologia após seis meses nos casos de ASC-US possivelmente não neoplásico e colposcopia em todos os casos de AGC (atipias glandulares) e ASC-H, atipias de células escamosas onde não se pode afastar lesão de alto grau. A colposcopia apresenta sensibilidade em torno de $96 \%{ }^{25}$.

Em relação ao tratamento proposto, $100 \%$ das NIC I ou HPV (LSIL) foram tratadas corretamente por exérese da lesão, visto que é o preconizado pelo Instituto Nacional de Câncer José Alencar Gomes da Silva (INCA), realizando o procedimento ambulatoriamente no CECON, referência do Estado do Acre. Estas devem 
continuar o seguimento citológico/colposcópico semestral por dois anos ambulatorialmente ${ }^{25}$.

De acordo com as Novas Diretrizes Brasileiras para o rastreamento do câncer do colo do útero, preconizadas pelo INCA no ano de 2011, as HSIL (NIC II e III) devem ser diretamente encaminhadas para colposcopia na unidade de média complexidade até 3 meses após o diagnóstico. Colposcopia positiva, com lesão restrita ao colo do útero, náo estendendo além do primeiro centímetro do canal, a conduta é a exérese da zona de transformação (EZT) pelo método ver-e-tratar. Quando ver-e-tratar não for possível, encaminhar a mulher para a unidade terciária (hospital) para tratamento, assim como HSIL que se estende à periferia do colo ou para a vagina ${ }^{24}$. Nesta pesquisa, $100,0 \%$ das NIC II e $93,8 \%$ das NIC III foram tratadas por exérese da lesão, e uma proporção pequena $(6,2 \%$ das NIC III) por histerectomia. As mulheres sem a possibilidade de tratamento por CAF ou com diagnóstico de câncer na biópsia devem ser tratadas pela histerectomia, conforme relatado anteriormente. Todas as pacientes que realizam exérese da lesão devem ser acompanhadas por dois anos e repetir o exame citológico após semestralmente ${ }^{25}$.

A conduta adotada para adenocarcinoma in situl invasor é a colposcopia imediata na unidade de média complexidade. Se a biópsia for negativa (sem invasão) deve-se proceder com a conização e se for positiva encaminhar para a unidade de alta complexidade ${ }^{25}$. As mulheres com câncer representaram $2 \%$ das alteraçóes citológicas e $42,1 \%$ foram tratadas por excérese da lesão por conização, $10,5 \%$ foram histerectomizadas e $47,4 \%$ por radioterapia e quimioterapia, conforme diagnóstico colposcópico que indicou cada tratamento. Nos casos em que for identificado adenocarcinoma in situ na peça resultante da conização, a mulher deve ser submetida à histerectomia simples ${ }^{25}$.

Lee et al. e o INCA sustentam que a conização pode ser considerada o tratamento definitivo para carcinoma micro invasor estádio IA1, independente de idade ou prole, podendo ser usada a eletrocirurgia ou lâmina fria. O envolvimento do espaço linfovascular é importante fator prognóstico para recorrência, e o comprometimento das margens da peça do cone define a necessidade de nova conização ou histerectomia ${ }^{24-25}$. No caso de mulheres com diagnóstico de câncer ou adenocarcinoma invasivo, devem ser encaminhadas para a unidade terciária para cirurgia. Todas as mulheres devem repetir o exame citológico após 6 meses $^{24}$.

Este estudo proporciona uma grande representatividade do rastreamento e tratamento do câncer do colo do útero no Estado do Acre, visto que $75 \%$ das mulheres acreanas utilizam o setor público de saúde. Pode-se observar o perfil vulnerável das mulheres com resultados colpocitológicos de ASCUS/AGC, LSIL e HSIL, além das limitaçōes dos serviços públicos em diagnosticar e tratar corretamente as atipias de significado indeterminado.

O presente estudo apresenta as limitaçóes dos estudos retrospectivos, onde se utilizam prontuários das pacientes disponíveis no CECON e nas unidades básicas de saúde, estes últimos nem sempre eram completos. Por outro lado, o presente estudo apresenta forças que precisam ser destacadas, dentre elas o fato de ter utilizado todos os casos de alteraçôes colpocitológicas diagnosticadas no SUS em Rio Branco (AC), Brasil, nos anos de 2007 e 2008. Considerando que $75 \%$ das mulheres desse município utilizam o serviço público, pode-se considerar que este é um estudo de base populacional e que nossos achados se aplicam a toda a população do município. No entanto, estudos prospectivos seriam na avaliação e monitorizaçáo do rastreamento e tratamento das atipias de significado indeterminado.

\section{CONCLUSÃO}

Este estudo evidenciou que na cidade de Rio Branco, somente $32,4 \%$ das mulheres estáo sendo tratadas e 13,6\% em tratamento expectante (LSIL e atipias), mostrando que, além de muitas não estarem recebendo tratamento, uma grande frequência recebe o diagnóstico citológico incorreto, fruto provavelmente de erros na coleta, leitura das lâminas citológicas. Dessa forma, sugere-se que sejam implementadas melhorias na qualidade do programa de prevenção do câncer do colo do útero do Estado do Acre, envolvendo a avaliação e controle do diagnóstico e tratamento, especialmente das mulheres diagnosticadas como ASCUS/AGC, para que se possa esperar alguma redução nas taxas de incidência e mortalidade por câncer do colo do útero nessa Região.

\section{AGRADECIMENTOS}

À Coordenação de Aperfeiçoamento de Pessoal de Nível Superior (CAPES) através da Associação Temporária estabelecida entre a Universidade Federal do Acre (Programa de Pós-Graduação em Saúde Coletiva); ao Programa de Pós-graduação em Saúde Pública e Meio Ambiente - Ensp/Fiocruz; e aos servidores do Centro de Controle em Oncologia do Acre (CECON/Acre), pelo auxílio para a execução desta pesquisa.

\section{CONTRIBUIÇÕES}

Patricia Rezende do Prado, Rosalina Jorge Koifman e Ilce Ferreira da Silva participaram da concepção, planejamento, obtenção, análise dos dados, redação e revisão final do estudo. Anna Luiza Moreira Santana participou da obtenção dos dados. 
Declaraçáo de Conflito de Interesses: Nada a Declarar.

\section{REFERÊNCIAS}

1. Ferlay J, Shin HR, Bray F, Forman D, Mathers C, Parkin DM. Estimates of worldwide burden of cancer in 2008: GLOBOCAN 2008. Int J Cancer. 2010;127(12):2893-917.

2. Jemal A, Bray F, Center MM, Ferlay J, Ward E, Forman D. Global cancer statistics. CA Cancer J Clin. 2011;61(2):69-90.

3. Instituto Nacional de Câncer José Alencar Gomes da Silva. Estimativa 2012: incidência de câncer no Brasil [Internet]. Rio de Janeiro: INCA; 2011 [citado 2012 jan 18]. 118 p. Disponível em: http://www.inca.gov.br/ estimativa/2012/estimativa20122111.pdf

4. Nakashima JP, Koifman S, Koifman RJ. Tendência da mortalidade por neoplasias malignas selecionadas em Rio Branco, Acre, Brasil, 1980-2006. Cad Saúde Pública. 2011;27(6):1165-74.

5. Castellsagué X, Díaz M, Sanjosé S, Muñoz N, Herrero R, Franceschi S, et al.; International Agency for Research on Cancer Multicenter Cervical Cancer Study Group. Worldwide human papillomavirus etiology of cervical adenocarcinoma and its cofactors: implications for screening and prevention. J Natl Cancer Inst. 2006;98(5):303-15.

6. Solomon D. The 1988 Bethesda System for reporting cervical/vaginal cytologic diagnoses. Developed and approved at the National Cancer Institute Workshop, Bethesda, Maryland, U.S.A., December 12-13, 1988. Acta Cytol. 1989;33(5):567-74.

7. Solomon D, Davey D, Kurman R, Moriarty A, O'Connor D, Prey M, et al. The 2001 Bethesda System: terminology for reporting results of cervical cytology. JAMA. 2002;287(16):2114-9.

8. Borges MFSO, Dotto LMG, Koifman RJ, Cunha MA, Muniz PT. Prevalência do exame preventivo de câncer do colo do útero em Rio Branco, Acre, Brasil, e fatores associados à não-realização do exame. Cad Saúde Pública. 2012;28(6):1156-66.

9. Mendonça VG, Lorenzato FRB, Mendonça JG, Menezes TC, Guimarães MJB. Mortalidade por câncer do colo do útero: características sociodemográficas das mulheres residentes na cidade de Recife, Pernambuco. Rev bras ginecol obstet. 2008;30(5):248-55.

10. Leal EAS, Leal Júnior OS, Guimarães MH, Vitoriano MN, Nascimento TL, Costa OLN. Lesōes precursoras do câncer de colo em mulheres adolescentes e adultas jovens do município de Rio Branco - Acre. Rev bras ginecol obstet. 2003;25(2):81-6.

11. Roteli-Martins CM, Longatto Filho A, Hammes LS, Derchain SFM, Naud P, Matos JC, et al. Associação entre idade ao início da atividade sexual e subsequente infecção por papilomavírus humano: resultados de um programa de rastreamento brasileiro. Rev bras ginecol obstet. 2007;29(11):580-7.

12. Burchell AN, Tellier PP, Hanley J, Coutlée F, Franco EL. Human papillomavirus infections among couples in new sexual relationships. Epidemiology. 2010;21(1):31-7.

13. Pinho AA, França Junior I, Schraiber LB, D’Oliveira AFPL. Cobertura e motivos para a realização ou não do teste de Papanicolaou no Município de São Paulo. Cad Saúde Pública. 2003;19(supl 2):S303-13.

14. Ng E, Wilkins R, Fung MF, Berthelot JM. Cervical cancer mortality by neighbourhood income in urban Canada from 1971 to 1996. CMAJ. 2004;170(10):1545-9.

15. Cuzick J, Sasieni P, Singer A. Risk factors for invasive cervix cancer in young women. Eur J Cancer. 1996;32A(5):836-41.

16. Martinelli PM. Tabagismo em adultos do município de Rio Branco - Acre: um estudo de base populacional [dissertação]. Rio Branco: Universidade Federal do Acre; 2012.

17. Simons AM, Phillips DH, Coleman DV. Damage to DNA in cervical epithelium related to smoking tobacco. BMJ. 1993;306(6890):1444-8.

18. Amorim VMSL, Barros MBA, César CLG, Carandina L, Goldbaum M. Fatores associados à não realização do exame de Papanicolaou: um estudo de base populacional no município de Campinas, São Paulo, Brasil. Cad Saúde Pública. 2006;22(11):2329-38.

19. Zeferino LC. O desafio de reduzir a mortalidade por câncer do colo do útero [editorial]. Rev bras ginecol obstet. 2008;30(5):213-5.

20. Kurman RJ, Solomon D. O Sistema Bethesda para o relato de diagnóstico citológico cervicovaginal. Rio de Janeiro: Revinter; 1997. 75 p.

21. Alves VAF, Lima MAN, Utagawa ML, Maeda MYS. Programa de controle de qualidade em citologia ginecológica do Instituto Adolfo Lutz: estratégias e análise crítica dos resultados de sua implantação piloto. AMB Rev Assoc Med Bras. 1991;37(1):36-42.

22. Westin MCA. Células glandulares atípicas e adenocarcinoma in situ de acordo com a classificação de Bethesda 2001: associação cito-histológica [dissertação]. Campinas: Universidade Estadual de Campinas; 2009.

23. Sebastião APM, Noronha L, Scheffel DLH, Garcia MJ, Carvalho NS, Collaço LM, et al. Estudo das atipias indeterminadas em relação à prevalência e ao percentual de discordância nos casos do Programa de Prevenção do Câncer Uterino do Paraná. J bras patol med lab. 2004;40(6):431-8.

24. Instituto Nacional de Câncer (Brasil). Diretrizes brasileiras para o rastreamento do câncer do colo do útero. Rio de Janeiro: INCA; 2011. 104 p.

25. Lee SW, Kim YM, Son WS, You HJ, Kim DY, Kim JH, et al. The efficacy of conservative management after conization in patients with stage IA1 microinvasive cervical carcinoma. Acta Obstet Gynecol Scand. 2009;88(2):209-15. 


\begin{abstract}
Introduction: Cervical cancer is the second most frequent cancer among women. This type of cancer is also the most common and the main cause of mortality among women in the North of Brazil, especially in the city of Rio Branco, Acre. Objective: To characterize the profile of women screened in the public health with cervical cytology results of ASCUS/AGC, LSIL e HSIL, between 2007 and 2008, in the city of Rio Branco / AC. Method: Cross-sectional study, with women diagnosed with cervical cancer precursor lesions identified by Pap smear and recorded in the Cervical Cancer Control Program in the years 2007 and 2008 in the Oncology Control Center in Acre - CECON/ AC. Results: $22.6 \%$ of women were under 25 years of age and $57.3 \%$ were between $25-45$ years, $89.1 \%$ were pardas, $59.8 \%$ had primary level education, $30.5 \%$ had the first sexual intercourse before the age of $14,64.9 \%$ had more than 3 children, $70.0 \%$ had more than two lifelong partners, $54.0 \%$ had not received treatment after cytologic changes diagnosis and $45.7 \%$ of cytologic diagnosis of atypical cells of undetermined significance were high-grade lesions or cancer on histopathology. Conclusion: Improvements are needed to enhance the quality of the cervical prevention program in the state of Acre including evaluation and control of diagnosis and treatment of cervical cancer, especially of women diagnosed as ASCUS/AGC. Such actions are expected to reduce the incidence and mortality from cervical cancer in this Region.

Key words: Uterine Cervical Neoplasms/prevention \& control; Uterine Cervical Neoplasms/therapy; Mass Screening; Cross-Sectional Studies
\end{abstract}

\title{
Resumen
}

Introducción: El cáncer del cuello del útero es el segundo cáncer más frecuente entre las mujeres. En el Norte de Brasil, especialmente en la ciudad de Río Branco / Acre, es el tipo de cáncer de más grande incidencia y mortalidad. Objetivo: Caracterizar el perfil de las mujeres examinadas con resultados de diferentes lesiones cervicales como ASCUS/AGC, LSIL y HSIL, entre el año 2007 a 2008, en el sector público en la ciudad de Río Branco / AC. Método: Estudio transversal, con las mujeres diagnosticadas con lesiones cervicales precursoras de cáncer identificados a través de la prueba Papanicolaou y registradas en el Programa de Control del Cáncer del cuello del útero en los años 2007 y 2008 en el Centro de Control en Oncología en Acre - CECON / AC. Resultados: el 22,6\% de las mujeres tenían menos de 25 ańos de edad y el 57,3\% tenían entre 25-45 años, 89,1\% tenían color pardo, el 59,8\% tenía estudios primarios, el 30,5\% tenían la primera relación sexual antes de los 14 años, 64,9\% tenían mas de 3 hijos, 70,0\% tuvieron más de dos parejas a lo largo de la vida, un 54,0\% no habían recibido tratamiento después del diagnostico citológicos anormal y el 45,7\% de los diagnósticos citológicos de atipias de significado indeterminado eran lesiones de alto grado o cáncer en el histopatológico. Conclusión: Se propone que sean implementadas las mejoras en la calidad del programa para prevenir el cáncer del cuello del útero en el Estado de Acre, involucrando la evaluación y control de diagnóstico y tratamiento, especialmente de las mujeres diagnosticadas como ASCUS / AGC, para que se pueda esperar una cierta reducción en la incidencia y la mortalidad por cáncer del cuello del útero en esta Región.

Palabras clave: Neoplasias del Cuello Uterino/prevención \& control; Neoplasias del Cuello Uterino/terapia; Tamizaje Masivo; Estudios Transversales 